\title{
Smoothing of ultrathin silver films by transition metal seeding
}

\author{
André Anders \\ Lawrence Berkeley National Laboratory, 1 Cyclotron Road, Berkeley, California 94720 \\ Eungsun Byon \\ Lawrence Berkeley National Laboratory, 1 Cyclotron Road, Berkeley, California 94720, \\ now at the Korean Institute of Machinery \& Materials, 66 Sangnam-dong, Changwon \\ 641-010, Korea \\ Dong-Ho Kim \\ Korean Institute of Machinery \& Materials, 66 Sangnam-dong, Changwon 641-010, \\ Korea \\ Kentaro Fukuda \\ Lawrence Berkeley National Laboratory, 1 Cyclotron Road, Berkeley, California 94720, \\ now at Nippon Sheet Glass Co. Ltd., Tsukuba, Ibaraki, 300-2635, Japan

\section{Sunnie H.N. Lim} \\ Lawrence Berkeley National Laboratory, 1 Cyclotron Road, Berkeley, California 94720, \\ now at the University of Sydney, School of Physics, A28, Sydney, NSW 2001, Australia
}

\begin{abstract}
.
The nucleation and coalescence of silver islands on coated glass was investigated by insitu measurements of the sheet resistance. Sub-monolayer amounts of niobium and other transition metals were deposited prior to the deposition of silver. It was found that in some cases, the transition metals lead to coalescence of silver at nominally thinner films with smoother topology. The smoothing or roughening effects by the presence of the transition metal can be explained by kinetically limited transition metal islands growth and oxidation, followed by defect-dominated nucleation of silver.
\end{abstract}




\section{Introduction}

Nucleation and growth are of great importance to numerous deposition processes and practical applications such as silver-based low emissivity coatings. For high transmission in the visible and high reflection in the infrared, it is desirable to utilize very thin silver films of low sheet resistance [1]. Very small thickness and low sheet resistance are clearly conflicting requirements demanding careful optimization of the growth process. Sheet resistance is determined by scattering of conduction electrons at defects, grain boundaries, and surfaces. Therefore it is desirable to produce ultrathin films with large aligned grains and very smooth surfaces. However, metal films tend to grow on oxides in Volmer-Weber (i.e., island) mode [2, 3]. To improve wetting and promote growth with preferential orientation it is common to coat glass with other transparent materials like $\mathrm{TiO}_{2}$ or even better with $\mathrm{ZnO}$ [4]. In application with tempered or bent glass, a "sacrificial” interlayer of a few nanometers thickness is used to protect silver from oxidation. The sacrificial material is oxidized in the process and becomes part of the transparent multilayer [5]. Using much thinner "sacrificial” films, we will demonstrate that the sacrificial material acts as seeds of nucleation for silver which, for certain coverage and material conditions, can lead to earlier silver coalescence with greatly reduced surface roughness.

\section{Experiments}

Two deposition methods were used: (i) filtered cathodic arc deposition, and (ii) magnetron sputtering. The setup for filtered cathodic arcs was described in earlier work on coalescence of silver islands [6] where no sacrificial material was used. In essence, 
each miniature arc source had a cathode rod of $6.25 \mathrm{~mm}$ diameter; the cathode is the feedstock for the metal plasma [7]. The sources (one for Ag, the other for the transition metal) operated in repetitively pulsed mode with arc pulses of $1.2 \mathrm{kA}$ amplitude, $600 \mu \mathrm{s}$ duration, and a repetition rate of 1.6 p.p.s. Each source had a $90^{\circ}$ magnetic filter (a curved, open solenoid) to remove unwanted metal debris ("macroparticles") from the plasma [8]. The chamber was cryogenically pumped to a base pressure of about $10^{-4} \mathrm{~Pa}$; no process gas was needed or used.

For the sputtering, 3” magnetrons were used. Silver and the transition metals were sputtered in DC mode (AE MDX 500 supply), whereas the $\mathrm{TiO}_{2}$ or $\mathrm{ZnO} 44 \mathrm{at} \% \mathrm{Al}$ underlayer on glass, if used, were sputtered in reactive "pulsed DC" mode using an AE Pinnacle Plus at $100 \mathrm{kHz}$ with $1 \mu$ s reverse time. The deposition rates were calibrated exsitu by measuring step heights of relatively thick films ( 100 nm) using a Dektak profilometer. The nominal thickness of ultrathin films was determined by counting arc pulses for the filtered arc process and by timing for the sputtering process after having determined that rates and thicknesses are indeed reproducible. For example, the deposition rate for the filtered arc process of silver was determined to be $0.023 \mathrm{~nm} / \mathrm{pulse}$, corresponding to about $1 / 10$ of a monolayer per pulse, while the instantaneous rate was $37 \mathrm{~nm} / \mathrm{s}$. For the DC sputtering process, a low power of only $50 \mathrm{~W}$ was selected leading to a deposition rate of $0.75 \mathrm{~nm} / \mathrm{s}$. Low power gave us better control of timing the process and the final nominal thickness. It should be stressed that the reported film thicknesses are nominal (they can be sub-monolayer or may consist of islands).

The substrates were uncoated standard microscope glass slides or slides precoated with reactively sputtered $\mathrm{TiO}_{2}$ or $\mathrm{ZnO}: 4 \mathrm{at} \% \mathrm{Al}$. The samples were cleaned with 
ethanol and deionized water, and dry-blown with nitrogen prior to being mounted on a water-cooled substrate holder. For both pre-coatings and silver coatings, the substrate temperature was generally near room temperature although the surface was subject to some heating by the deposition process.

The films were evaluated by in-situ sheet resistance measurements and atomic force microscopy (AFM, Nanoscope IIIa). As in our earlier paper [6], the samples were prepared for in-situ sheet resistance measurements by masked deposition of two silver contact pads such that a square area of $2.5 \mathrm{~cm} \times 2.5 \mathrm{~cm}$ was formed between them. Therefore, the measurement of resistance between the pads directly indicated the sheet resistance. The interface resistance between film and contact pad was neglected with the argument that film and contact material were the same (silver).

\section{Results}

When the nominal thickness of the growing film between the contact pads exceeded a certain thickness, e.g. $8 \mathrm{~nm}$, a sharp drop of the sheet resistance by several orders of magnitude can be observed, indicating the coalescence of islands (Fig. 1). The resistance was measured by either a Keithley 177 or by FLUKE 189 precision multimeter. Possible effects of the measuring current on the result were excluded by repeating the measurements with the instrument disconnected during the deposition process and controlling that same final sheet resistance was obtained.

The original objective of the experiments aimed to investigate niobium as a sacrificial material. An initially surprising result was found when using unusually thin films. Nominally $0.1 \mathrm{~nm}$ thick $\mathrm{Nb}$ film had a much greater modifying effect than a 
nominally $0.2 \mathrm{~nm}$ thick film (Fig. 1). Repetition of the experiment confirmed that this observation was reproducible.

AFM measurements revealed that nominal $0.1 \mathrm{~nm}$ of $\mathrm{Nb}$ led to much smoother, fine-grained silver films than $0.2 \mathrm{~nm}$ of $\mathrm{Nb}$ (Fig. 2). The RMS roughness was about the same for the samples without and with $0.2 \mathrm{~nm} \mathrm{Nb}(2.9-3.2 \mathrm{~nm})$, and much reduced for the $0.1 \mathrm{~nm} \mathrm{Nb}$ samples (only $0.8 \mathrm{~nm}$ ). Detailed information on roughness and grain size can be found in Table 1.

The experiments were continued focusing on the industrially more relevant sputtering method. When using very small amounts of niobium, nominal 0.1 and $0.2 \mathrm{~nm}$, coalescence of silver occurred earlier as compared to deposition without this material, however, $1 \mathrm{~nm}$ of $\mathrm{Nb}$ had the opposite effect. We found again the same effect when switching to $\mathrm{Ti}$ as the seeding layer material (Fig. 3).

Further experiments with $\mathrm{Ni}, \mathrm{Cr}, \mathrm{Zr}$, Ta, and Mo showed that the effect on coalescence was not always the same, not even qualitatively. Details for those materials will be described elsewhere; here we only mention that for some materials, such as Cr, coalescence of silver islands was delayed, i.e. occurred at greater nominal silver thickness, even when very small amounts of the seed material were used.

\section{Discussion}

To understand why small amounts of transition metals affect the growth of silver in a very different fashion, depending on surface coverage and kind of material, one should consider the thermodynamic driving forces that determine two or three dimensional growth. Minimization of Gibbs free energy in a thin film system requires 
considering the metal-oxide interfacial free energy and the metal surface free energy. The growth mode under thermodynamic equilibrium conditions is determined by

$$
\Delta \gamma=\gamma_{\text {metal }}+\gamma_{\text {metal-substrate }}-\gamma_{\text {substrate }}
$$

where $\gamma_{\text {metal }}, \gamma_{\text {metal-substrate }}$, and $\gamma_{\text {substrate }}$ are the free energies of metal surfaces, metal-oxide substrate interfaces, and the oxide substrate surfaces, respectively. If $\Delta \gamma>0$, 3D island growth occurs (Volmer-Weber mode); otherwise, 2D layer growth is thermodynamically preferred (this is known as Bauer's criterion [9]). The 2D growth mode may be continued (Frank - van der Merve mode), or 3D islands may growth on top of the initial 2D layers (Stranski-Kastranov mode) because the surface of the original substrate is shielded by the initial layers.

Studying $\mathrm{TiO}_{2}(110)$-metal systems, $\mathrm{Hu}$ and coworkers [10] have shown that the issue of island versus layer growth modes can be classified via the relation of the heat of formation of metal oxides and the heat of sublimation of metals, i.e., parameters representing the strength of metal-oxide interfacial interactions and the strength of metal/metal lateral interactions, respectively. In their Fig. 4, Hu and coworkers [10] showed that noble metals (e.g., Ag, Au, Pt) grow on $\mathrm{TiO}_{2}$ in $3 \mathrm{D}$ island mode while alkali and other less noble metals (e.g., $\mathrm{Na}, \mathrm{Ba}, \mathrm{Al}$ ) grow in 2D layer mode, and most transition metals are in a transition zone where both 3D or 2D growth could occur.

So far, the arguments are based on thermodynamic driving forces which work well for systems near thermodynamic equilibrium. For experiments at relatively low temperature, like room temperature as in our case, kinetic limitations may prevent the most stable structures from being produced (for example, see temperature effects on growth of Bi thin films on glass [11]). Nucleation and film growth cannot always be 
described by one of the three "classical" growth modes but other, kinetically limited modes may apply.

Campbell studied the kinetically limited 2D island (2 DI) growth mode which is characterized by the formation of 2D (monolayer) islands reaching significant critical coverage (15-85\%), which is defined as the coverage before the onset of bilayer and multilayer growth. The monolayer islands are not thermodynamically stable: when the temperature is increased, the metal atoms irreversibly form tall islands and reduce the coverage. Based on careful studies using LEED (low energy electron diffraction) and other techniques, Campbell [3] found that at the beginning of the deposition, individual atoms are deposited that are relatively mobile which allows them to meet and to form monolayer islands. When the critical coverage is reached, island growth switches to 3D (bilayer and multilayer) growth. This island "thickening" depends in particular on the kind of metal. Alkali and early transition metals tend to form large monolayer islands and reach high coverage whereas mid-to-late transition metals as well as noble metals have a thermodynamic tendency to cluster into 3D islands on oxides. In the latter case, the critical coverage can be very low [3].

The early transition metals have the highest affinity to oxygen. To consider whether or not they can reduce the oxide substrate from a thermodynamic point of view, one could check that the heat of the reducing reaction is greater than the heat of formation $\Delta H^{0}$ of the newly formed oxide (both normalized to one mol of molecular oxygen). For example, the titanium dioxide surface can be reduced according to [3]

$$
2 \mathrm{TiO}_{2}(\mathrm{~s}) \rightarrow \mathrm{Ti}_{2} \mathrm{O}_{3}(\mathrm{~s})+1 / 2 \mathrm{O}_{2}(\mathrm{~g})
$$


with the heat of the reducing reaction $\Delta H^{0}=2 \Delta H_{\mathrm{Ti}_{2} \mathrm{O}_{3}}-4 \Delta H_{\mathrm{TiO}_{2}}=736 \mathrm{~kJ} / \mathrm{mol}$ (per mol of oxygen). This needs to be compared to the heat of reaction of

$$
\mathrm{Me}(s)+2 \mathrm{TiO}_{2}(s) \rightarrow \mathrm{MeO}(s)+\mathrm{Ti}_{2} \mathrm{O}_{3}(s)
$$

where $\mathrm{Me}$ stands for the metal deposited. In the case of $\mathrm{Nb}$, the heat of formation is $\Delta H_{\mathrm{Nb}_{2} \mathrm{O}_{3}}^{0}=623 \mathrm{~kJ} / \mathrm{mol}$ (per mol of oxygen) [12], hence the reduction of the solid oxide by $\mathrm{Nb}$ is thermodynamically not preferred. For $\mathrm{ZnO}$, the other main based layer material used in various experiments, the situation is similar: the heat of formation of $\mathrm{ZnO}$ is 688 $\mathrm{kJ} / \mathrm{mol}$ (per mol of oxygen) [13], closer to but still greater than the heat of formation of $\mathrm{Nb}_{2} \mathrm{O}_{3}$.

However, oxidation of the seed metal is very likely because the experiments were not done in UHV (ultrahigh vacuum). The previously discussed findings in the literature were based on either computation or experiments with carefully prepared surfaces in UHV. In high vacuum (HV), the main source of oxygen is the residual gas, which mainly consists of water vapor. The propensity for oxidation is much greater due to the abundance of oxygen from sources other than the solid oxide substrate. In our experiment, silver was deposited about 1 minute after depositing the nominal submonolayer of transition metal. Although the vacuum chamber was not vented between steps, the time between the deposition steps was sufficient to provide a relatively "intense" bombardment of the transition metal with residual gas molecules. Therefore, one can assume that the transition metal is fully oxidized by the time silver deposition started.

The differences seen in our experiment affecting silver growth are based on the difference in the seeds provided for nucleation. Their size, shape, distribution, material, 
and oxidation state depends on the seed material; most likely these five factors cannot be reduced to a single number comparing the different transition metals. The LEED experiments in UHV are a good guide for what might occur, namely, in our kinetically limited growth at room temperature, the 3D (Volmer-Weber) or the 2DI mode may be dominant, depending on the material. Additionally, oxidation is very likely, which will inhibit the mobility of the transition metals. Oxidized atoms, clusters, 2D, and 3D islands provide the necessary attraction for mobile silver adatoms. They are the nucleation centers. It is reasonable to assume that the island size and thickness were quite different for the different nominal thicknesses, like the $0.1 \mathrm{~nm}$ and $0.2 \mathrm{~nm}$ of niobium (oxide). The latter are more likely to be taller islands, especially when considering the kinetically limited 2DI mode. In any case investigated, the density of seed islands (“defects") was high for the deposition of silver: it is likely that silver growth occurred by defectdominated nucleation and therefore was so strongly affected by the presence of the transition metal (oxide) islands. This qualitative picture calls for more detailed measurements, especially of the topology of the surface between the transition metal and silver deposition steps.

\section{Conclusions}

In summary, we have studied the effect of small amounts of transition metal on the nucleation and coalescence of silver films. Onset of silver coalescence and surface roughness was found to be very sensitive to the kind and amount of seed material. Submonolayer amounts (nominally $0.1 \mathrm{~nm}$ ) of some transition metals (especially with $\mathrm{Nb}$ and Ti) lead to significant smoothing and coalescence at thinner silver thickness. If more 
than the equivalent of a monolayer was deposited ( $0.2 \mathrm{~nm}$ and more), greater roughness and coalescence at greater thickness was observed. The observations can qualitatively be explained in a thermodynamic model of kinetically limited transition metal island growth followed by oxidation: the formation of initially 2D and later 3D islands is possible, depending on the material and other factors.

\section{Acknowledgements}

E. Byon, K. Fukuda and S.H.N Lim acknowledge support by the post-doctoral fellowship program of the Korea Research Foundation (KRF), the Nippon Sheet Glass Co. Ltd, and the American Australian Association for the 2004 ANZ Fellowship, respectively. The authors thank M.P. Delplancke-Ogletree for helpful comments. This work was supported by the Assistant Secretary for Energy Efficiency and Renewable Energy, Office of Building Technology, of the U.S. Department of Energy under Contract No. DE-AC02-05CH11231. 


\section{References}

[1] H. J. Gläser, Large Area Glass Coating. Dresden, Germany: Von Ardenne Anlagentechnik GmbH, 2000.

[2] M. Ohring, Materials Science of Thin Films. Deposition and Structure, 2nd ed. San Diego: Academic Press, 2002.

[3] C. T. Campbell, Surface Science Reports 27 (1997) 1.

[4] R. Dannenberg, D. Glenn, and E. Stach, "Microstructural comparison of Ag films grown on amorphous $\mathrm{TiO}_{2}$ and polycrystalline $\mathrm{ZnO}$,” 44th Annual Technical Conference Proceedings, Society of Vacuum Coaters, Philadelphia, 2001, 218-224.

[5] M. Arbab, Thin Solid Films 381 (2001) 15.

[6] E. Byon, T. H. Oates, and A. Anders, Appl. Phys. Lett. 82 (2003) 1634.

[7] R. A. MacGill, M. R. Dickinson, A. Anders, O. R. Monteiro, and I. G. Brown, Rev. Sci. Instrum. 69 (1998) 801.

[8] A. Anders, Surf. Coat. Technol. 120-121 (1999) 319.

[9] E. Bauer, Zeitschr. für Kristallographie 372 (1957) 110.

[10] M. Hu, S. Noda, and H. Komiyama, Surface Science 513 (2002) 530.

[11] D.-H. Kim, S.-H. Lee, J.-K. Kim, and G.-H. Lee, Appl. Surf. Sci. 252 (2006) 3525.

[12] O. Kubaschewski and B. E. Hopkins, Oxidation of Metals and Alloys, 2rd edition ed. London: Butterworth, 1962.

[13] O. Kubaschewski and E. L. Evans, Metallurgical Thermochemistry, Reprint of 3rd edition ed. Oxford: Pergamon Press, 1965. 


\section{Figure Captions}

FIG. 1 Sheet resistance of filtered arc deposited silver films on $\mathrm{TiO}_{2}$ coated glass

substrate. Very small amounts of Nb promote the onset of silver coalescence.

FIG. 2 AFM pictures ( $1 \mu \mathrm{m} \times 1 \mu \mathrm{m})$ of $25-\mathrm{nm}$ silver films deposited by filtered arc on $\mathrm{TiO}_{2}$ coated glass without $\mathrm{Nb}$ (top), with nominal $0.1 \mathrm{~nm} \mathrm{Nb}$ (center), and with nominal $0.2 \mathrm{~nm} \mathrm{Nb}$ (bottom). The z-axis units are $50 \mathrm{~nm} / \mathrm{div}$ (top and bottom) and $40 \mathrm{~nm} / \mathrm{div}$ (center).

FIG. 3 Evolution of the sheet resistance of silver films on $\mathrm{ZnO}$ :4at\%Al with sputtered Ti as the seeding layer material. The large “jumps” are caused by a very raid fall of the resistance during growth due to the coalescence of conducting silver islands. The limited sampling rate (1 point per second) of the data acquisition system was not adequate to show this change with many data points. 


\section{Table Caption}

Table 1. Average and root-mean-square (rms) roughness, maximum peak heights, and grain size for $25 \mathrm{~nm}$ silver layers formed on titanium-oxide-coated glass without and with $\mathrm{Nb}$ underlayer of $0.1 \mathrm{~nm}$ and $0.2 \mathrm{~nm}$ thickness. The grain size was determined from 2D-

AFM images of the same samples shown in Fig. 2, using image-processing software. 


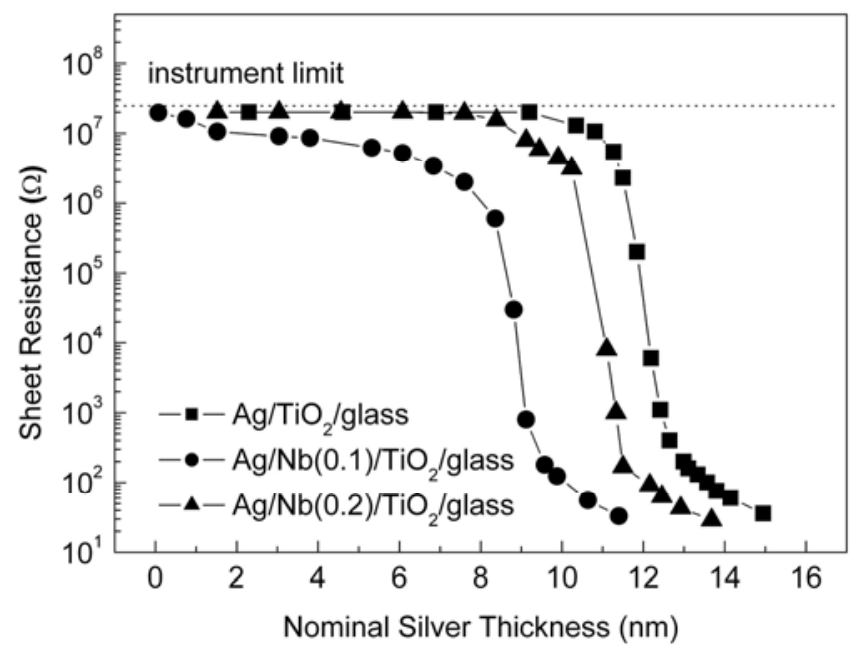

Fig. 1 

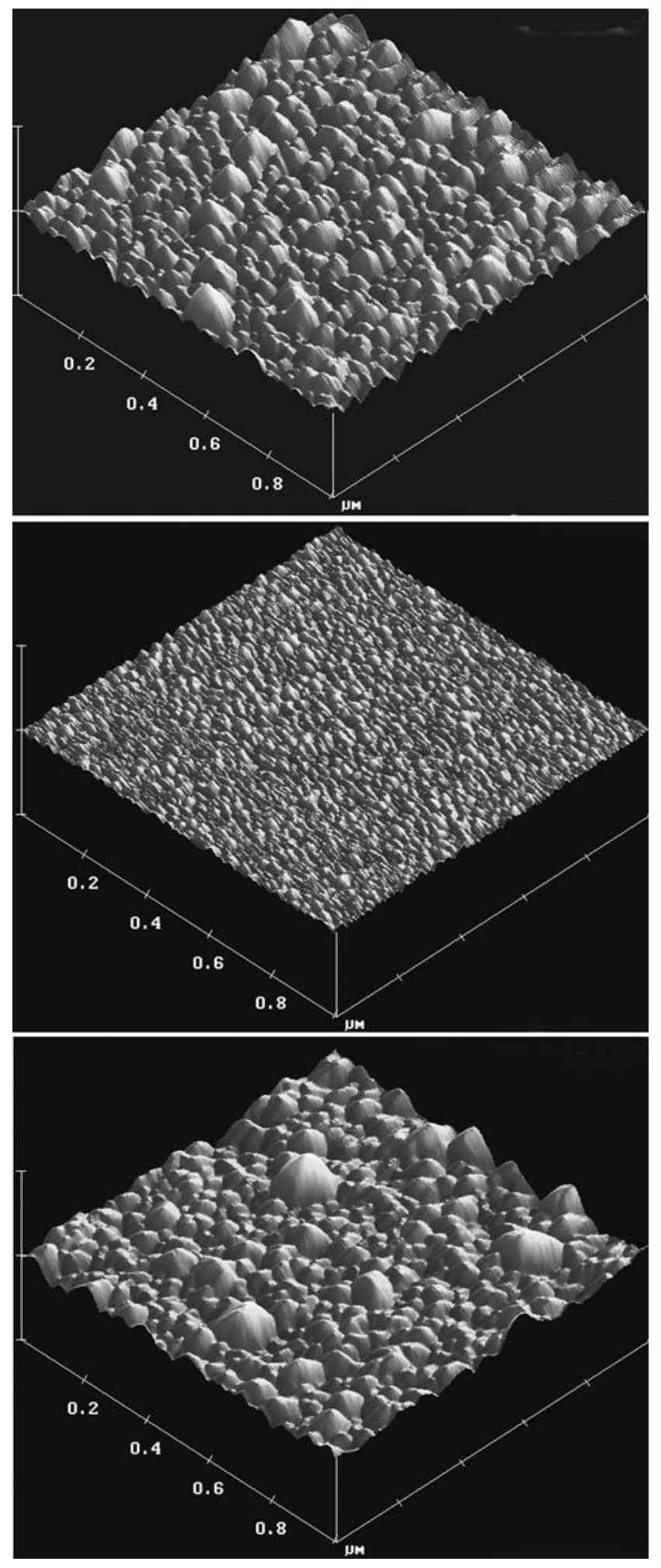

Fig. 2 


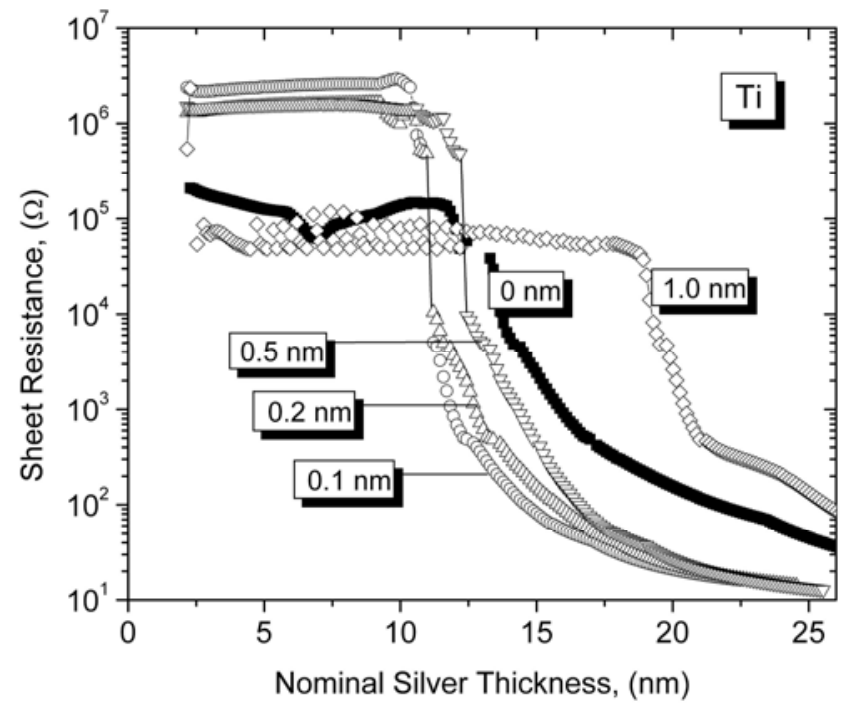

Fig. 3 